\title{
Saddle Points of the Complementary Error Function
}

\author{
By Henry E. Fettis, James C. Caslin and Kenneth R. Cramer
}

Abstract. The first one hundred zeros of the derivative of the function $w(z)=e^{-z^{2}}$ $\operatorname{Erfc}(-i z)$ are given, together with an asymptotic formula for estimating the higher zeros.

1. In a previous paper by the present authors [1], the zeros of the function (1)

$$
w(z)=e^{-z^{2}} \operatorname{Erfc}(-i z)
$$

were obtained. In this paper, the values of $z=x+i y$ for which

$$
d w / d z=0
$$

are given. These points represent singular points of the family of curves

$$
\phi(x, y) \equiv|w|=\text { const }
$$

in the $x-y$ plane since at such a point the direction $d y / d x$ of these curves is undefined. As in the case of the zeros of $w(z)$, the saddle points lie in the lower half-plane and are symmetrically located with respect to the $y$-axis. For convenience, we introduce the function $Y(\rho)=(\sqrt{ } \pi / 2) w(i \rho)$, which satisfies the differential equation

$$
d Y / d \rho=2 \rho Y-1 .
$$

Thus, at a saddle point, $\rho=\rho_{n}$,

$$
2 \rho_{n} Y\left(\rho_{n}\right)=1 .
$$

With the aid of the differential equation (4), we can expand $Y$ in the vicinity of a saddle point as a Taylor series, viz.,

$$
Y=+\frac{1}{2 \rho_{n}}+\frac{1}{2 \rho_{n}}\left(\rho-\rho_{n}\right)^{2}+\frac{1}{3}\left(\rho-\rho_{n}\right)^{3}+\cdots .
$$

Hence

$$
\frac{1}{Y}=2 \rho_{n}-2 \rho_{n}\left(\rho-\rho_{n}\right)^{2}-\frac{4 \rho_{n}^{2}}{3}\left(\rho-\rho_{n}\right)^{3}+\cdots
$$

Introducing the variable $t=\rho-1 / 2 Y$, this may be written

$$
\begin{aligned}
t & =\left(\rho-\rho_{n}\right)+\rho_{n}\left(\rho-\rho_{n}\right)^{2}+\frac{2 \rho_{n}^{2}}{3}\left(\rho-\rho_{n}\right)^{3}+\cdots \\
& =\left(\rho-\rho_{n}\right)+\rho\left(\rho-\rho_{n}\right)^{2}-\left[1-\frac{2 \rho^{2}}{3}\right]\left(\rho-\rho_{n}\right)^{3}+\cdots .
\end{aligned}
$$

Received June 26, 1972.

AMS (MOS) subject classifications (1970). Primary 33A20. 


\section{Therefore}

$$
\rho-\rho_{n}=t-\rho t^{2}+\left[1-8 \rho^{2} / 3\right] t^{3}+\cdots,
$$

or

$$
\rho_{n}=\rho-t+\rho t^{2}-\left[1-8 \rho^{2} / 3\right] t^{3}+\cdots .
$$

Equation (3) may also be expressed in terms of $Y$ as follows:

$$
\rho_{n}=\frac{1}{2 Y}\left[1+t^{2}+\frac{4}{3 Y} t^{3}+\cdots\right] .
$$

The above series will converge rapidly if $\rho$ is close to a saddle point $\rho_{n}$. In the next section, an asymptotic approximation to the saddle points is derived which may be used as a first approximation. By computing the corresponding values of $Y$ and $t$ and substituting these into Eq. (11), an improved approximation to $\rho_{n}$ is obtained. If necessary, ${ }^{*}$ the process may be repeated using the newly computed value of $\rho$, and continued until convergence is reached. A sample calculation leading to the first saddle point is given at the end of the next section.

2. Asymptotic Approximation to the Saddle Points. At a saddle point, we have, from Eq. (5), $2 \rho Y=1$ or

$$
w=+i / \pi^{1 / 2} z .
$$

The saddle points are assumed to be of the form $z=x-i y$, with $x>0, y>0$. Setting $w(x+i y)=u+i v$, Eq. (12) is equivalent to

$$
2 e^{y^{2}-x^{2}} e^{2 i x y}-u+i v=i / \pi^{1 / 2} z .
$$

Replacing $w$ by the first three terms of the continued fraction gives

$$
u-i v=-\frac{i}{\pi^{1 / 2}}\left[\frac{z^{2}-1}{z\left(z^{2}-3 / 2\right)}\right],
$$

and Eq. (13) becomes

$$
2 e^{y^{2}-x^{2}} e^{2 i x y} \doteq \frac{-i}{\pi^{1 / 2}}\left\{\frac{1}{z\left[2 z^{2}-3\right]}\right\} .
$$

Since $\arg (z)=-\pi / 4+\sigma$, it follows that the argument of the right side of (15) is $\pi / 4-\sigma$. Hence,

$$
2 x y=\left(2 n+\frac{1}{4}\right) \pi+\beta,
$$

where $0 \leqq \beta \leqq \pi / 2$ and since, asymptotically, $x \doteq y$, we take, as the limiting value of $x$ and $y$,

$$
\lambda=\left(\left(n+\frac{1}{8}\right) \pi\right)^{1 / 2}
$$

and set**

* By computing a sufficient number of additional terms in Eq. (11), only one application would be required.

** For the justification of this form, see [1, Eq. (29)]. 


$$
x=\lambda+\alpha+p, \quad y=\lambda-\alpha+p .
$$

From Eq. (16) we have, equating magnitudes,

$$
\begin{aligned}
2 e^{-4 \lambda \alpha-4 \alpha p} & \doteq \frac{1}{2 \sqrt{ } \pi\left(x^{2}+y^{2}\right)^{3 / 2}}=\frac{1}{2^{5 / 2} \sqrt{ } \pi\left[\lambda^{2}+\alpha^{2}+2 \lambda p\right]^{3 / 2}} \\
& =\frac{1}{2^{5 / 2} \sqrt{ } \pi \lambda^{3}\left[1+2 p / \lambda+(\alpha / \lambda)^{2}\right]^{3 / 2}} .
\end{aligned}
$$

Hence

$$
\begin{aligned}
2 e^{-4 \lambda \alpha} & =1 / 2^{5 / 2} \pi^{1 / 2} \lambda^{3} ; \\
\alpha & \doteq \ln \left(128 \pi \lambda^{6}\right) / 8 \lambda .
\end{aligned}
$$

The value of $p$ is determined by equating arguments in Eq. (15). We find, denoting the argument of the right side by $\phi$,

$$
\begin{aligned}
\tan 2 x y & \doteq 1+4 \alpha^{2}-8 \lambda p \\
\tan \phi & \doteq 1-6 \alpha / \lambda+3 / 2 \lambda^{2} .
\end{aligned}
$$

This gives

$$
p \doteq\left(8(\lambda \alpha)^{2}-12(\lambda \alpha)+3\right) / 16 \lambda^{3} \text {. }
$$

Thus, the desired asymptotic approximation to three terms is

$$
\left\{\begin{array}{c}
x \\
-y
\end{array}\right\}=\lambda \pm \frac{1}{8 \lambda} \ln \left(128 \pi \lambda^{6}\right)+\frac{\frac{1}{8}\left[\ln \left(128 \pi \lambda^{6}\right)\right]^{2}-\frac{3}{2} \ln \left(128 \pi \lambda^{6}\right)+3}{16 \lambda^{3}} .
$$

The use of the approximation (25) in conjunction with Eq. (11) is illustrated below for the first saddle point. Equation (17) with $n=1$ gives

$$
\lambda=1.8799712060
$$

and this, when substituted into Eq. (25), gives

$$
x \doteq 2.5332619139, \quad y \doteq-1.2321384069 \text {. }
$$

The corresponding value of $Y$ is

$$
Y=-.0766358650+.1594090127 i
$$

Thus

$$
t=-.0073085147+.0144867658 i \text {. }
$$

Substituting in Eq. (11) the values of $t$ and $y$ as given by Eqs. (28) and (29), we arrived at the improved values

$$
x \doteq 2.5471305433, \quad y \doteq-1.2251557198,
$$

the corresponding values of $Y$ and $t$ being

$$
\begin{aligned}
Y & =-.07667898752+.1594172691 i \\
t & =.00000137615-.00000251508 i
\end{aligned}
$$




\begin{tabular}{|c|c|c|c|c|c|}
\hline \multicolumn{6}{|c|}{ Zeros of $w^{\prime}(z)$} \\
\hline $\mathbf{N}$ & $x$ & $Y$ & $\mathbf{N}$ & $x$ & $Y$ \\
\hline $\begin{array}{r}1 \\
2 \\
3 \\
4 \\
5 \\
6 \\
7 \\
8 \\
9 \\
10\end{array}$ & 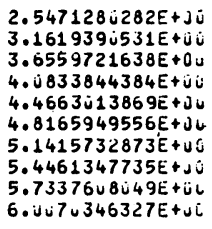 & $\begin{array}{l}-1.2251570959 E+O O \\
-2 . i 2559613 U 7 E+O U \\
-2.6288721547 E+U U \\
-3.1323514518 E+U O \\
-3.5728492 U 33 E+O U \\
-3.969 U 173280 E+O U \\
-4.3318395797 E+U C \\
-4.6684220832 E+O O \\
-4.983671135 U E+O U \\
-5.2811402113 E+O U\end{array}$ & $\begin{array}{l}51 \\
52 \\
53 \\
54 \\
55 \\
56 \\
57 \\
58 \\
59 \\
60\end{array}$ & $\begin{array}{l}1.2883628281 E+C 1 \\
1.30055 u 374 E+C 1 \\
1.3126239378 E+U 1 \\
1.3245864838 E+U 1 \\
1.3364414869 E+U 1 \\
1.3481906248 E+U 1 \\
1.3598378492 E+U 1 \\
1.3713853935 E+U 1 \\
1.38283578 v 2 E+U 1 \\
1.3941914268 E+U 1\end{array}$ & $\begin{array}{l}-1.2464770326 E+01 \\
-1.2589548699 E+01 \\
-1.2713113798 E+01 \\
-1.2835500701 E+C 1 \\
-1.2956742377 E+C 1 \\
-1.3076870288 E+01 \\
-1.3195914484 E+01 \\
-1.3313903689 E+01 \\
-1.3430865384 E+01 \\
-1.3546825878 E+01\end{array}$ \\
\hline $\begin{array}{l}11 \\
12 \\
13 \\
14 \\
15 \\
16 \\
17 \\
18 \\
19 \\
20\end{array}$ & $\begin{array}{l}6.2679376653 E+J i \\
6.518 u 3 i 2553 E+j u \\
6.7585678528 E+J u \\
6.9945787939 E+J U \\
7.2149182 u 67 E+j u \\
7.4323 u 64525 E+J i \\
7.6433572151 E+J U \\
7.8485984629 E+J U \\
8.04848838 U 8 E+U i \\
8.2434276772 E+J U\end{array}$ & $\begin{array}{l}-5.5634993766 E+U U \\
-5.832814312 U E+U O \\
-6 . U 9 C 7215155 E+O U \\
-6.3385432717 E+O C \\
-6.5773658881 E+O U \\
-6.8 \cup 8 \cup 945823 E+U O \\
-7 . ن 314930 \cup 11 E+O U \\
-7.2482123176 E+O O \\
-7.458813 J 776 E+O U \\
-7.6637818847 E+O O\end{array}$ & $\begin{array}{l}61 \\
62 \\
63 \\
64 \\
65 \\
66 \\
67 \\
68 \\
69 \\
70\end{array}$ & $\begin{array}{l}1.4054546519 E+U 1 \\
1.4166276813 E+U 1 \\
1.4277126525 E+v 1 \\
1.4387116197 E+i 1 \\
1.4496265587 E+v 1 \\
1.46 \cup 45937 \cup 3 E+U 1 \\
1.4712118848 E+01 \\
1.4818858656 E+U 1 \\
1.492483612 J E+U 1 \\
1.5030 ن 49632 E+U 1\end{array}$ & $\begin{array}{l}-1.3661810379 E+01 \\
-1.3775843054 E+01 \\
-1.3888947393 E+01 \\
-1.4001144760 E+01 \\
-1.4112457444 E+01 \\
-1.4222945709 E+01 \\
-1.4332509334 E+01 \\
-1.4441287357 E+01 \\
-1.4549258111 E+01 \\
-1.4656439265 E+01\end{array}$ \\
\hline $\begin{array}{l}21 \\
22 \\
23 \\
24 \\
25 \\
26 \\
27 \\
28 \\
29 \\
30\end{array}$ & 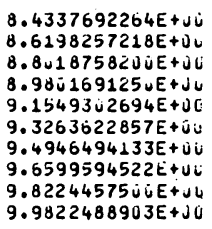 & 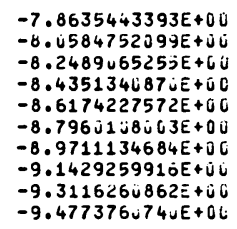 & $\begin{array}{l}71 \\
72 \\
73 \\
74 \\
75 \\
76 \\
77 \\
78 \\
79 \\
86\end{array}$ & $\begin{array}{l}1.5134533006 E+U 1 \\
1.523829551 j E+U 1 \\
1.5341351892 E+U 1 \\
1.544371640 J E+U 1 \\
1.55454 \cup 2812 E+U 1 \\
1.5646424452 E+U 1 \\
1.5746794214 E+i 1 \\
1.584652458 j E+U 1 \\
1.5945627637 E+U 1 \\
1.6044115098 E+U 1\end{array}$ & $\begin{array}{l}-1.4762847849 E+C 1 \\
-1.4868500294 E+01 \\
-1.4973412456 E+01 \\
-1.5077599645 E+01 \\
-1.5181076652 E+01 \\
-1.5283857772 E+01 \\
-1.5385956828 E+01 \\
-1.548738719 U E+01 \\
-1.5588161800 E+01 \\
-1.5688293186 E+01\end{array}$ \\
\hline $\begin{array}{l}31 \\
32 \\
33 \\
34 \\
35 \\
36 \\
37 \\
38 \\
39 \\
40\end{array}$ & $\begin{array}{l}1 . j 139498135 E+J 1 \\
1 . u 294312663 E+v 1 \\
1 . v 4468 i 2641 E+J 1 \\
1 . u 59707 J 15 j E+j 1 \\
1 . u 7452 i 9996 E+v 1 \\
1 . v 89131412 E+j 1 \\
1.1 \cup 35453688 E+v 1 \\
1.11777167 .9 E+v 1 \\
1.1318171446 E+j 1 \\
1.1456885382 E+j 1\end{array}$ & 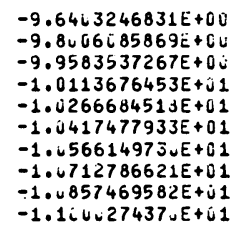 & $\begin{array}{l}81 \\
82 \\
83 \\
84 \\
85 \\
86 \\
87 \\
88 \\
89 \\
90\end{array}$ & $\begin{array}{l}1.6141998312 E+U 1 \\
1.6239288287 E+U 1 \\
1.6335995699 E+\cup 1 \\
1.64321369 j 5 E+01 \\
1.6527703961 E+U 1 \\
1.6622724632 E+01 \\
1.67172024 \dot{1} 1 E+U 1 \\
1.6811146485 E+[1 \\
1.6904565840 E+U 1 \\
1.6997469177 E+C 1\end{array}$ & $\begin{array}{l}-1.5787793485 E+01 \\
-1.5886674456 E+01 \\
-1.5984947497 E+01 \\
-1.6082623664 E+01 \\
-1.6179713679 E+01 \\
-1.6276227949 E+01 \\
-1.6372176576 E+01 \\
-1.6467569372 E+01 \\
-1.6562415867 E+01 \\
-1.6656725323 E+01\end{array}$ \\
\hline $\begin{array}{l}41 \\
42 \\
43 \\
44 \\
45 \\
40 \\
47 \\
48 \\
49 \\
60\end{array}$ & 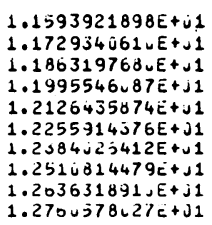 & 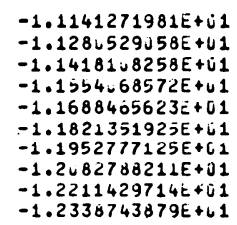 & $\begin{array}{r}91 \\
92 \\
93 \\
94 \\
95 \\
96 \\
97 \\
98 \\
99 \\
100\end{array}$ & 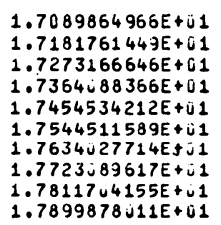 & $\begin{array}{l}-1.6750506743 E+C 1 \\
-1.6843758883 E+C 1 \\
-1.6936520258 E+01 \\
-1.7028769153 E+01 \\
-1.7124523634 E+01 \\
-1.7211791552 E+01 \\
-1.73 \cup 2580552 E+01 \\
-1.7392898083 E+C 1 \\
-1.7482751401 E+01 \\
-1.7572147579 E+U 1\end{array}$ \\
\hline
\end{tabular}

This leads to the next approximation

$$
x \doteq 2.5471280282, \quad y \doteq-1.2251570959 .
$$

which is now correct to eleven figures, the error being $O\left(t^{4}\right)$.

Aerospace Research Laboratories Wright-Patterson Air Force Base Ohio 45433

1. Henry E. Fettis, James C. Caslin \& Kenneth R. Cramer, "Complex zeros of the error function and of the complementary error function," Math. Comp., v. 27, 1973, pp. 401407. 